\title{
Cognitive Radios with Ergodic Capacity Guarantees for Primary Users
}

\author{
Antonio G. Marques, Member, IEEE, Luis M. Lopez-Ramos, Student Member, IEEE, and Javier Ramos
}

\begin{abstract}
Cognitive radios implement adaptive resource allocation schemes that exploit knowledge of the channel state information to optimize the performance of the secondary users while limiting the interference to the primary users. The algorithms in this paper are designed to maximize the weighted sum-rate of secondary users which transmit orthogonally and adhere to three different constraints: i) limits on the long-term (average) power at each secondary transmitter; ii) limits on the long-term interfering power at each primary receiver; and iii) limits on the long-term capacity loss inflicted to each primary receiver. Although the long-term capacity constraints render the resultant optimization problem non-convex, it holds that it has zero-duality gap and that, due to the favorable structure in the dual domain, it can be efficiently solved.
\end{abstract}

Index Terms-Cognitive radios, resource management, nonlinear optimization.

\section{INTRODUCTION}

The perceived spectrum under-utilization along with the proliferation of new wireless services have motivated recent research on dynamic spectrum management and wireless cognitive radios (CRs) which are capable of sensing and accessing the spectrum dynamically [3], [10], [8]. Secondary users (SUs) in the CR adapt their transmission to limit the interference to the primary user (PU) receivers which hold the licence of the frequency band. To carry out these tasks, the CR needs to sense not only the gain of the secondary network links, but also the channels between secondary transmitters and primary receivers. The information of secondary links allows SUs to mitigate fading and take advantage of good channel realizations, while the information of primary links guarantees that interference is kept under control. Based on the measurements obtained through sensing, SUs will adapt their available resources (here, power, rate and scheduling coefficients) to the channel conditions. The merits of adaptive schemes which exploit knowledge of statistical and instantaneous channel state information (CSI) to optimally allocate the transmit resources in traditional wireless systems are well documented; see [2, Chap. 9]. However, for channeladaptive schemes to be deployed in CR scenarios, several challenges not present in traditional wireless networks need to be considered. Among the most important design challenges we find that instantaneous CSI (especially that of the primary network) is difficult to acquire [11], [17] and that the adaptive schemes need to satisfy additional constraints to keep the interference low [10], [8]. Different alternatives have been

All authors are with the Dep. of Signal Theory and Communications, King Juan Carlos University, Madrid, Spain. Emails: see http://www.tsc.urjc.es

This work is supported by the Spanish Ministry of Science and Innovation proposed in the literature to cope with the latter challenge. For example, to keep interference under control some works limit the power of the interference at the primary receiver side, either by imposing instantaneous (short-term) or average (long-term) interference power constraints; see, e.g., [12], [11], [16]. The latter are better suited for fading channels because they can exploit the diversity of the interfering link [12]. Other designs use a probabilistic approach to limit the probability of interfering the primary transmissions (outage probability); see, e.g., [6], [15], [17].

Motivated by these findings, we design resource allocation (RA) algorithms that optimize the communication performance of the SUs and limit the interference to the PUs. We focus on underlay CRs where SUs adapt their power and rate loadings dynamically, and access orthogonally a set frequency bands which are primarily devoted to PU transmissions. Orthogonal here means that if a SU is transmitting, no other SU can be active in the same band. The RA schemes are then obtained as the solution of a weighted sum-average rate maximization subject to three types of constraints: i) limits on the long-term (average) power transmitted by each SU; ii) limits on the long-term interfering power at each PU [16]; and iii) limits on the long-term capacity loss inflicted to each PU. Consideration of iii) is challenging because the interfering (SU) powers render the capacity term non-convex, and it is the main contribution of this work. In spite of being non-convex, it holds that the formulated problem has zero duality gap. As a result, the Langrangian relaxation is optimal. Additionally, the operating conditions of the secondary network (and the formulation of the objective to optimize) are such that the problem in the dual domain can be separated across users and frequency bands. This favorable structure allows for a significant reduction on the complexity required to find the optimal solution and, hence, renders the non-convex problem computationally tractable. To facilitate exposition, the algorithms are designed under the assumption of perfect CSI. The changes required to account for CSI imperfections are briefly discussed at the end of the manuscript.

The rest of the paper is organized as follows. Section II presents the model for the CSI, describes the operating conditions of the secondary network, and formulates the rules that secondary transmissions must obey to limit the interference to PUs. Section III deals with the design of the optimal RA schemes. The end of that section is devoted to discuss how the schemes have to be modified to account for other meaningful operating conditions. Numerical simulations and conclusions 
in Sections IV and V wrap-up this paper. ${ }^{1}$

\section{MODEL DESCRIPTION}

We consider a CR with $M$ SUs (indexed by $m$ ) transmitting opportunistically and orthogonally over $K$ different frequency bands (indexed by $k$ ). For simplicity, we assume that: i) each band has the same bandwidth and is occupied by a different PU; and ii) the secondary network has an access point (AP) which is the destination of all SUs. The AP acts as a central scheduler which collects the CSI and then makes the RA decisions. Extensions to scenarios where those assumptions do not hold true can be handled with a moderate increase of complexity.

\section{A. Channel state information}

In a CR scenario, the AP collects not only the information of the SU-to-SU links, but also the information of the SUto-PU links. For simplicity, we will develop our schemes under the assumption that the CSI is perfect. The required modifications when the CSI contains imperfections will be briefly discussed at the end of Section III. For notational purposes, the channel's instantaneous power gain between the $m$ th SU and the AP in the $k$ th frequency band at instant $n$ is denoted by $h_{k, 2}^{m}[n]$ (noise-normalized squared magnitude of the fading coefficient). Subscript " 2 " is used to emphasize that the channel pertains to secondary receivers. Similarly, $h_{k, 1}^{m}[n]$ denotes the instantaneous normalized power gain between the $m$ th SU and the $k$ th PU at instant $n$. Here, subscript " 1 " is used for primary receivers. The overall CSI ( $2 M K$ instantaneous gains) will be denoted as $\mathbf{h}[n]$.

\section{B. Resources at the secondary network}

Now, we introduce the variables to be designed, i.e, the variables that will be adapted as a function of the (primary and secondary) CSI $\mathbf{h}[n]$. Let $w_{k, 2}^{m}$ denote a boolean variable such that $w_{k, 2}^{m}=1$ if the $m$ th $\mathrm{SU}$ is scheduled to transmit into the $k$ th band and $w_{k, 2}^{m}=0$ otherwise. Provided that $w_{k, 2}^{m}=1$, let $p_{k, 2}^{m}$ denote the instantaneous power transmitted over the $k$ th band by the $m$ th SU. Under bit error rate or capacity constraints, instantaneous rate and power variables are coupled. This rate-power coupling will be represented by the function $r_{k, 2}^{m}\left(h_{k, 2}^{m} p_{k, 2}^{m}\right)$. Throughout this paper it is assumed that the rate-power function is given by Shannon's capacity formula $\log _{2}\left(1+h_{k, 2}^{m} p_{k, 2}^{m}\right)$. However, the fundamental results in this paper hold for any increasing and concave rate-power function.

The secondary CR operates in a time-block fashion, where the duration of each block corresponds to the coherence time of the fading channel. This way, at every time $n$ the AP will use the current CSI vector $\mathbf{h}$ to find the (optimum) value of $w_{k, 2}^{m}$ and $p_{k, 2}^{m}$. Since $\mathbf{h}$ depends on $n$ and $\left\{w_{k, 2}^{m}, p_{k, 2}^{m}\right\}$ depend on $\mathbf{h}$, the value of the design variables $\left\{w_{k, 2}^{m}, p_{k, 2}^{m}\right\}$ will clearly

\footnotetext{
${ }^{1} N$ otation: ${ }^{T}$ denotes vector transposition; $x^{*}$ the optimal value of variable $x ; \mathbb{E}[\cdot]$ expectation; $\wedge$ the boolean "and" operator; $\mathbb{1}_{\{\cdot\}}$ the indicator function $\left(\mathbb{1}_{\{x\}}=1\right.$ if $x$ is true and zero otherwise); and $[x]_{a}^{b}$ the projection of the scalar $x$ onto the $[a, b]$ interval, i.e., $[x]_{a}^{b}:=\min \{\max \{x, a\}, b\}$.
}

vary across time. Through the manuscript, we will write $\mathbf{h}$, $w_{k, 2}^{m}(\mathbf{h})$ and $p_{k, 2}^{m}(\mathbf{h})$, or $\mathbf{h}[n], w_{k, 2}^{m}[n]$ and $p_{k, 2}^{m}[n]$, wherever is convenient to emphasize the corresponding dependence.

Once the variables are introduced, we formulate constraints that these variables need to satisfy. To ensure that at most one user transmits into a given band $k$, we need

$$
\sum_{m} w_{k, 2}^{m}(\mathbf{h}) \leq 1, \quad \forall k .
$$

If the left hand side (LHS) of the constraint is equal to one, then one user is accessing the channel (orthogonal access). If it is equal to zero, then none is transmitting (either because all secondary channels are poor, or because it causes very high interference to the PUs). We also consider that the maximum average (long-term) power the $m$ th SU can transmit is $\check{p}_{2}^{m}$; hence,

$$
\mathbb{E}\left[\sum_{k} w_{k, 2}^{m}(\mathbf{h}) p_{k, 2}^{m}(\mathbf{h})\right] \leq \check{p}_{2}^{m}, \quad \forall m
$$

Such a constraint is not only reasonable to effect QoS across SUs, but also to limit the power consumption of each of the SU transmitters. The expectation in (2) is taken over all possible values of $h_{k, 1}^{m}[n]$ and $h_{k, 2}^{m}[n]$; i.e, considering all $m, k$, and $n$. While (1) needs to hold for each and every channel realization (hence for each and every time instant), (2) only needs to hold in the long-term.

\section{Cognitive constraints}

The next step is to identify the rules that dictate how CR transmissions affect the performance of the PUs. Such rules will be formulated as constraints that will be incorporated into the optimization problem that gives rise to the RA schemes. In other words, the cognitive constraints will represent how SUs have to modify their behavior so that the damage caused to the PUs is kept under control.

When the cognitive constraints are formulated, there are several issues that have a significant impact both in terms of the operation of the $\mathrm{CR}$ and the mathematical formulation of the optimization problem. Two of the most important are discussed next. The first factor is whether the interference constraints are formulated as instantaneous (short-term) or as average (long-term) constraints. The former requires the constraint to hold for each and every time instant, while the latter requires the constraint to hold on average (taking into account all time instants jointly). Clearly, instantaneous constraints are more restrictive than their average counterparts (which can exploit the so-called "cognitive diversity" of the primary CSI [12], [11]), and therefore the performance of the secondary network will be higher in the latter case [16]. Mathematically, long-term constraints are typically dualized, while short-term constraints are handled using alternative methods. The second factor is the metric used to measure the damage that the CRs inflict to the PUs. Among the metrics considered in the literature we find: interfering power at the PUs, probability on interfering the PUs, and rate loss inflicted to the PUs. Most works in the literature have focused on limiting the interfering power. The reason is twofold: i) it is a simple (and intuitive) metric to measure the interference, and ii) it can be formulated as a convex constraint. Limiting the rate loss may 
be considered a better alternative because it focuses on the actual damage that the interference causes to the PUs (most communications systems are designed to either guarantee or maximize a certain transmission rate). From a mathematical perspective, constraints limiting the rate loss are typically nonconvex. As a result, fewer works have explored that alternative. The problem of limiting the probability of interference for a system with operating conditions very similar to the ones considered in this paper was thoroughly investigated in [17].

As already mentioned, the main contribution of this work is to limit the long-term rate (capacity) loss experienced by the PUs. However, we will also impose limits on the long-term interfering power [16]. Joint consideration of rate loss and interfering power constraints will help us to better understand the similarities and differences between these two alternatives.

We start with the formulation of the long-term interfering power constraints. Let $\check{p}_{k, 1}$ denote the maximum average interfering power the $k$ th primary receiver can tolerate and recall that the $m$ th SU transmits in the $k$ th channel only if the boolean scheduling variable $w_{k, 2}^{m}(\mathbf{h})$ is equal to one. Then, the following $K$ constraints need to hold

$$
\mathbb{E}\left[\sum_{m} w_{k, 2}^{m}(\mathbf{h}) h_{k, 1}^{m} p_{k, 2}^{m}(\mathbf{h})\right] \leq \check{p}_{k, 1}, \quad \forall k .
$$

The fact that the expectation is taken across all possible channel realizations reflects that (3) is a long-term constraint. Clearly, for a given channel realization $\mathbf{h}$, at most one of the $M$ terms inside the expectation is active. This property will be exploited in upcoming sections.

Next, we formulate the long-term capacity constraints. For such a purpose, we define the function $r_{k, 1}(x):=$ $\log _{2}\left(1+\frac{\gamma_{k, 1}}{1+x}\right)$, where $\gamma_{k, 1}$ and $x$ stand for the normalized signal-to-noise ratio (SNR) and the interfering power at the $k$ th PU receiver, respectively. Our formulation will guarantee a minimum long-term rate of $\check{r}_{k, 1}$ for the $k$ th PU. This minimum rate can be either a fixed value [4], or expressed as a percentage of the rate that the PU achieves when no SUs are present. Mathematically, the rate requirement in the latter case can be written as $\check{r}_{k, 1}:=\left(1-\check{\varepsilon}_{k}\right) \mathbb{E}\left[r_{k, 1}(0)\right]$ where $\check{\varepsilon}_{k} \in(0,1)$ is the maximum (relative) rate loss that the SUs can cause to the $k$ th PU. With these issues in mind, the long-term capacity constraint is formulated as

$$
\begin{aligned}
& \mathbb{E}\left[\sum_{m} w_{k, 2}^{m}(\mathbf{h}) r_{k, 1}\left(h_{k, 1}^{m} p_{k, 2}^{m}(\mathbf{h})\right)\right] \\
& \quad+\mathbb{E}\left[\left(1-\sum_{m} w_{k, 2}^{m}(\mathbf{h})\right) r_{k, 1}(0)\right] \geq \check{r}_{k, 1}, \quad \forall k .
\end{aligned}
$$

The first term (which is itself the summation of $M$ terms) represents the capacity of the $k$ th $\mathrm{PU}$ when there is a SU transmitting in the $k$ th channel. The second term represents the case of no SU using the $k$ th channel. As in the case of constraint (3), for a given channel realization $\mathbf{h}$ only one of the $M+1$ terms inside the expectation is active. This property will be very important in reducing the computational complexity required to find the optimal RA. The expression in (4) also confirms that if the constraint is written as $f\left(p_{k, 2}^{m}(\mathbf{h})\right) \leq 0$, then $f(\cdot)$ is a non-convex function.

\section{FORMULATING AND SOLVING THE RESOURCE ALLOCATION PROBLEM}

To formulate the optimization problem that gives rise to the optimum RA algorithms, we need to identify: i) the variables to be optimized, ii) the constraints the variables need to satisfy, and iii) the metric to be optimized. The first step was accomplished in Section II-B. Regarding the second step, boolean variables $w_{k, 2}^{m}(\mathbf{h})$ are constrained to belong to the set $\{0,1\}$ and variables $p_{k, 2}^{m}(\mathbf{h})$ are constrained to belong to the set $\left[0, \check{p}_{k, 2}^{m}\right]$, where $\check{p}_{k, 2}^{m}$ represents an upperbound on the short-term transmit-power (peak power constraints). Moreover, $w_{k, 2}^{m}(\mathbf{h})$ and $p_{k, 2}^{m}(\mathbf{h})$ need to satisfy the constraints in (1) and (2) and those in (3) and (4).

Regarding the third step (metric to be optimized), we are interested in maximizing the weighted sum-average rate (capacity) given by $\bar{c}_{2}:=\sum_{k, m} \mathbb{E}\left[\beta^{m} w_{k, 2}^{m}(\mathbf{h}) r_{k, 2}^{m}\left(h_{k, 2}^{m} p_{k, 2}^{m}(\mathbf{h})\right)\right]$, where $\beta^{m}>0$ represents a user-dependent priority coefficient. Other objective functions such as sum-utility rate could be used without changing the basic structure of the solution; see, e.g., [4], [14] for further details.

Under all previous considerations, the optimal RA is obtained as the solution of the following problem:

$$
\begin{aligned}
& \max _{\left\{w_{k, 2}^{m}(\mathbf{h}), p_{k, 2}^{m}(\mathbf{h})\right\}} \bar{c}_{2} \\
& \text { s. to : } \quad w_{k, 2}^{m}(\mathbf{h}) \in\{0,1\}, 0 \leq p_{k, 2}^{m}(\mathbf{h}) \leq \check{p}_{k, 2}^{m}, \quad(1),
\end{aligned}
$$$$
\text { (2), (3), (4); }
$$

where the dependence of the optimization variables on the CSI $\mathbf{h}$ has been made explicit. Note that (5a) is the (longterm) objective we are interested in optimize, $(5 \mathrm{~b})$ collects all the short-term constraints the RA needs to satisfy, and $(5 \mathrm{c})$ collects all long-term constraints. As we will see in the next section, the approach to handle (5b) and (5c) will be different.

\section{A. Optimal resource allocation}

The main challenge to find the optimal RA is that (5) is not a convex problem. Basically, there are three sources of non-convexity in (5): i) scheduling coefficients $w_{k, 2}^{m}$ are constrained to belong to $\{0,1\}$, which is a non-convex set; ii) the monomials $w_{k, 2}^{m} p_{k, 2}^{m}, w_{k, 2}^{m} r_{k, 1}^{m}$ and $w_{k, 2}^{m} r_{k, 2}^{m}$ are not jointly convex; and iii) the constraint (4) is not convex with respect to (w.r.t.) $p_{k, 2}^{m}$. The two first sources on non-convexity can be "easily" bypassed by transforming (relaxing) the problem in (5) into a convex one which yields the same optimality conditions; see, e.g., [13]. However, the third source of nonconvexity can not be bypassed. Two undesirable consequences associated with lack of convexity are [1]: (c1) zero-duality gap is not guaranteed, and (c2) development of numerical algorithms that find the optimal solution in polynomial time is not guaranteed either. Remarkably, it can be shown that for the optimization at hand: the problem in (5) exhibits zeroduality gap $^{2}$. This result implies that the constraints can be dualized without losing optimality [1]. However, (c2) still

\footnotetext{
${ }^{2}$ The basic idea to show that the duality gap is zero is that the source of non-convexity comes from a constraint of the form $\mathbb{E}_{\mathbf{x}}[g(\mathbf{y}, \mathbf{x})]$, where $g(\mathbf{y}, \mathbf{x})$ is a non-convex function w.r.t. $\mathbf{y}$, and $\mathbf{x}$ is a random process with infinite support [cf. (4)]. We refer the reader to [5] for further details.
} 
holds, so that finding an efficient algorithm to optimize the (unconstrained) Lagrangian is still challenging. Interestingly, due to the structure of (5) we will show that the optimization can be separated (decomposed) across channels and users, decreasing dramatically the computational complexity of finding the optimal solution.

After the previous discussion, we are ready to present the solution of (5). Our approach to deal with the constraints in (5) is twofold. The long-term constraints (2), (3) and (4) in (5c) will be dualized, while the constraints in (5b) (all shortterm) will be handled using alternative methods such as scalar projections. Regarding the long-term constraints, let $\pi^{m}, \theta_{k}$ and $\rho_{k}$ denote the Lagrange multipliers associated with (2), (3) and (4), respectively. With this notational conventions, it can be shown (proof is omitted due to space limitations) that the optimal solution of (5) is

$$
\begin{aligned}
& \varphi_{k}^{m}\left(p_{k, 2}^{m}[n]\right):=\beta^{m} r_{k, 2}^{m}\left(h_{k, 2}^{m}[n] p_{k, 2}^{m}[n]\right) \\
& \text { - } \pi^{m} p_{k, 2}^{m}[n] \\
& \text { - } \theta_{k} h_{k, 1}^{m}[n] p_{k, 2}^{m}[n] \\
& +\quad \rho_{k} r_{k, 1}\left(h_{k, 1}^{m}[n] p_{k, 2}^{m}[n]\right) \\
& p_{k, 2}^{m *}[n]:=\left[\arg \max _{p_{k, 2}^{m}[n]} \varphi_{k}^{m}\left(p_{k, 2}^{m}[n]\right)\right]_{0}^{\check{p}_{k, 2}^{m}} \\
& w_{k, 2}^{m *}[n]:=\mathbb{1}_{\left\{\left(m=\arg \max _{l} \varphi_{k}^{l}\left(\left(p_{k}^{l *}[n]\right)\right)\right) \wedge\left(p_{k, 2}^{m *}[n]>0\right)\right\}}
\end{aligned}
$$

Key for understanding the solution of (5) is the definition of the functional $\varphi_{k}^{m}(\cdot)$ in (6). Mathematically, $\varphi_{k}^{m}(x)$ represents the contribution to the Lagrangian of (5) if the transmit power is $p_{k, 2}^{m}[n]=x$ and $w_{k, 2}^{m}[n]=1$. Intuitively, (6) can be interpreted as a user-channel quality indicator (the higher the indicator, the better). Under this interpretation, the rates of PUs and SUs are rewards (first and fourth terms), and the transmit and interfering powers are costs (second and third terms). The corresponding prices are $\beta^{m}, \rho_{k}, \pi^{m}$ and $\theta_{k}$, respectively. The indicator also manifests the existing trade-off between the SUs (first and second terms) and the PUs (third and fourth terms).

Based on the definition $\varphi_{k}^{m}\left(p_{k, 2}^{m}[n]\right)$, equation (7) reveals that $p_{k, 2}^{m *}[n]$ is found separately for each of the user-channel pairs. Similarly, (8) reveals that to find $\left\{w_{k, 2}^{m *}[n]\right\}_{m=1}^{M}$, i.e., the optimal scheduling for channel $k$; no information from channels other than $k$ is required. These attractive features are present because the optimization problem in the dual domain is separable across users and channels (see [14], [17]). Keys for this property to hold are the consideration of orthogonal access in the secondary network and the definition of the objective in (5).

We now analyze in further detail the optimal RA. Starting with the optimal scheduling in (8), we observe that $w_{k, 2}^{m *}[n]$ is available in closed form (provided that the optimum power is known). Equation (8) reveals that the scheduling follows a winner-takes-all strategy, guaranteeing that the access is orthogonal (at most one user is active), opportunistic ( $\varphi_{k}^{m}$ is a continuous random variable), and greedy (only the user with highest quality in a given band must be scheduled). The details of the optimum power allocation are a bit more intricate. To obtain $p_{k, 2}^{m *}[n]$ we need first to maximize $\varphi_{k}^{m}\left(p_{k, 2}^{m}[n]\right)$ w.r.t. $p_{k, 2}^{m}[n]$. Consider first a simplified case where the CR constraints (3) and (4) are not present. In such a case only the two first terms in (6) are present, so that $\varphi_{k}^{m}(\cdot)$ is strictly concave and differentiable (the first term is strictly concave and differentiable and the second is linear). As a result, the optimization is convex and $p_{k, 2}^{m *}[n]$ can be easily found. Specifically, $p_{k, 2}^{m *}[n]$ for this case is available in closed form as $p_{k, 2}^{m *}[n]=\left[\frac{\beta^{m} \log _{2}(\exp (1))}{\pi_{m}}-\frac{1}{h_{k, 2}^{m}}\right]_{0}^{\check{p}_{k, 2}^{m}}$. The previous expression is basically a waterfilling power loading [2] projected onto the feasible interval defined by the instantaneous constraints. When the CR constraint (3) is active, the third term in (6) needs to be considered. However, since that term is linear w.r.t. $p_{k, 2}^{m}[n]$, the structure of $\varphi_{k}^{m}(\cdot)$ is basically the same and $p_{k, 2}^{m *}[n]$ can still be efficiently found. In fact, the solution follows again a (modified) waterfilling scheme $p_{k, 2}^{m *}[n]=\left[\frac{\beta^{m} \log _{2}(\exp (1))}{\pi_{m}+\theta_{k} h_{k, 1}^{m}[n]}-\frac{1}{h_{k, 2}^{m}}\right]_{0}^{\check{p}_{k, 2}^{m}}$; see, e.g., [16]. However, when all four terms in (6) are considered, the optimization is challenging because $\varphi_{k}^{m}(\cdot)$ is not concave any more. The reason is that the last term is strictly convex, rendering the sum of the four terms in (6) non-concave and therefore, the optimization non-convex.

However, the fact of the optimization not being convex does not necessarily imply that $p_{k, 2}^{m *}[n]$ can not be efficiently found. The first reason is that optimizing $\varphi_{k}^{m}(\cdot)$ involves a single (scalar) variable. As a result, simple exhaustive search related methods (which are known to be very inefficient if the dimensionality of the search space is medium-high) can be used. The second reason is that the structure of $\varphi_{k}^{m}(\cdot)$ can be exploited to focus the search on a small searching region. For example, it can be rigorously shown that the waterfilling solution is an upperbound for $p_{k, 2}^{m *}[n]$. Moreover, it can also be easily shown that if the CSI is perfect (which is the case considered in this paper), then $\varphi_{k}^{m}(\cdot)$ has at most three stationary points. This readily implies that $p_{k, 2}^{m *}[n]$ is either zero or one of those three points. Once $\left\{p_{k, 2}^{m *}[n]\right\}_{m=1}^{M}$ are found, finding $\left\{w_{k, 2}^{m *}[n]\right\}_{m=1}^{M}$ just requires the evaluation of closed-form expressions [cf. (8)]. In other words, because in the dual domain the problem can be separated across users and channels, optimizing the Lagrangian does not require solving one non-convex problem in a $2 M K$ dimensional space. Rather, $M K$ closed forms (for the scheduling coefficients) and $M K$ non-convex problems in a one dimensional space (for the power loadings) need to be solved. Recall that the key factors for the problem being separable in the dual domain were: i) the fact of considering orthogonal access for the SUs; ii) the definition of the metric to be optimized as a summation across users; and iii) the fact that the long-term constraints were dualized.

Remark 1: The RA schemes have been developed under the assumption that the PUs are always active. However, they can be easily modified to account for scenarios where that assumption does not hold true. For that purpose, let $a_{k}[n]$ be a boolean variable which is one if the $k$ th $\mathrm{PU}$ is active at time $n$ and zero otherwise. Then, our formulation needs to be modified as follows. All the terms in the cognitive constraints inside the expectations must be multiplied by $a_{k}[n]$, so that the specific constraint only needs to hold for instants $n$ (CSI realizations) for which $a_{k}[n]=1$. Moreover, for the optimal allocation to account for these modifications, the third and 
fourth terms in (6), which are the ones accounting for the PUs, must be multiplied by $a_{k}[n]$ too. Mathematically, this implies that

$$
\begin{aligned}
\varphi_{k}^{m}\left(p_{k, 2}^{m}[n]\right) & :=\beta^{m} r_{k, 2}^{m}\left(h_{k, 2}^{m}[n] p_{k, 2}^{m}[n]\right) \\
& -\pi^{m} p_{k, 2}^{m}[n] \\
& -\theta_{k} a_{k}[n] h_{k, 1}^{m}[n] p_{k, 2}^{m}[n] \\
& +\rho_{k} a_{k}[n] r_{k, 1}\left(h_{k, 1}^{m}[n] p_{k, 2}^{m}[n]\right) .
\end{aligned}
$$

Remark 2: The RA schemes have been developed under the assumption that the CSI is perfect. This assumption can be unrealistic in practical deployments. For example, the CSI of the SU-to-SU links is typically quantized and the knowledge of the SU-to-PU may be noisy and outdated (because the channels are not sensed at every time instant). Such imperfections render the knowledge of $\mathbf{h}[n]$ at instant $n$ probabilistic. Thanks to the favorable structure of our problem in the dual domain, our formulation can account for these imperfections by redefining the link indicator in (6) as

$$
\begin{aligned}
\varphi_{k}^{m}\left(p_{k, 2}^{m}[n]\right) & :=\beta^{m} \mathbb{E}_{h_{k, 2}^{m}[n]}\left[r_{k, 2}^{m}\left(h_{k, 2}^{m}[n] p_{k, 2}^{m}[n]\right)\right] \\
& -\pi^{m} p_{k, 2}^{m}[n] \\
& -\theta_{k} \mathbb{E}_{h_{k, 1}^{m}[n]}\left[h_{k, 1}^{m}[n]\right] p_{k, 2}^{m}[n] \\
& +\rho_{k} \mathbb{E}_{h_{k, 1}^{m}[n]}\left[r_{k, 1}\left(h_{k, 1}^{m}[n] p_{k, 2}^{m}[n]\right)\right] .
\end{aligned}
$$

Note that the expectations in the previous expression: i) are taken over a scalar random variable; and ii) must be carried over the instantaneous channel distribution [17]. In other words, over the probabilistic description of $h_{k, 1}^{m}[n]$ and $h_{k, 2}^{m}[n]$ available at instant $n$. For example, if the CSI is quantized and we know that at instant $n$ the gain $h_{k, 2}^{m}[n]$ falls into the quantization region $\mathcal{R}$, then the expectations $\mathbb{E}_{h_{k, 2}^{m}[n]}[\cdot]$ will be computed averaging only over the realizations of $h_{k, 2}^{m}[n]$ that fall into (belong to) region $\mathcal{R}$.

Remark 3: Different methods can be used to set the value of $\pi^{m}, \theta_{k}$ and $\rho_{k}$. Traditionally, $\left\{\pi^{m}, \theta_{k}, \rho_{k}\right\}$ are set to a constant value $\left\{\pi^{m *}, \theta_{k}^{*}, \rho_{k}^{*}\right\}$ corresponding to the value that maximizes the dual function associated with (5) (recall that the duality gap is zero). This implies that if $\pi^{m}=\pi^{m *}$, $\theta_{k}=\theta_{k}^{*}$ and $\rho_{k}=\rho_{k}^{*}$ are substituted into (6)-(8), the resulting RA is indeed the optimal solution of (5) [1]. The main drawback associated with this approach is that $\left\{\pi^{m *}, \theta_{k}^{*}, \rho_{k}^{*}\right\}$ need to be found through iterative numerical search which, at every iteration, requires averaging over all possible states of $\mathbf{h}$ (including channel imperfections). Recently, alternative approaches that rely on stochastic approximation tools have been proposed to find the value of the multipliers [4], [7]. These approaches do not try to find the optimal value of $\left\{\pi^{m *}, \theta_{k}^{*}, \rho_{k}^{*}\right\}$, but an estimate of it $\pi^{m}[n], \theta_{k}[n]$ and $\rho_{k}[n]$ which is updated at every time instant and remains sufficiently close to $\left\{\pi^{m *}, \theta_{k}^{*}, \rho_{k}^{*}\right\}$. Such approaches have advantages that are especially attractive in CR setups, namely: i) they are robust to channel non-stationarities (which are common in environments with interference); ii) they do not need to have statistical knowledge of the channels; and iii) they can cope with changes in either the secondary network (number of users, QoS levels) or primary network (limits on the interfering power, rate loss or capacity function of the PUs). In other words, the stochastic schemes are useful to learn the environment on-the-fly and keep track of it [17].

\section{NUMERICAL SIMULATIONS}

The default simulation setup is the following: $M=5, K=$ $10, \beta^{m}=1, \check{p}_{2}^{m}=1, \check{p}_{k, 2}^{m}=40, \check{p}_{k, 1}=0.3$, and $\varepsilon_{k}=$ 0.10. The amplitudes of the SU-to-PU and SU-to-SU links are Rayleigh distributed, so that $h_{k, 1}^{m}[n]$ and $h_{k, 2}^{m}[n]$ follow an exponential distribution. The average SNR for all SU-to-SU is $3 \mathrm{~dB}$. The average SNR for all SU-to-PU is 0dB. The SNR for all PU-to-PU links is $\gamma_{k}=10 \mathrm{~dB}$.

Seven RA schemes are tested: S1) the optimal scheme that maximizes the performance of the SUs and ignores all cognitive constraints; S2) the optimal scheme in this paper setting $\check{p}_{k, 1}=\infty$ (i.e., ignoring the long-term interfering power constraint); S3) the optimal scheme in this paper setting $\check{\varepsilon}_{k}=1$ (i.e., ignoring the long-term capacity loss constraint); S4) the optimal scheme in this paper; S5) the scheme in S4 but replacing the long-term interfering power constraint with an instantaneous one (i.e., setting the peak constraint as $\left.\check{p}_{k, 2}^{m}=\check{p}_{k, 1} / h_{k, 1}^{m}[n]\right)$; S6) the scheme in S4 but replacing the long-term rate loss constraint with an instantaneous one; and S7) the scheme in S4 but replacing the long-term interfering power and rate loss constraints with their instantaneous counterparts.

For these seven schemes, Tables I-III list the values of the: average power transmitted by the SUs (denoted as $\bar{p}_{2}$ ), average weighted sum-capacity of the SUs $\left(\bar{c}_{2}\right)$, average interfering power at the PUs (denoted as $\bar{p}_{1}$ ), average capacity transmitted by the PUs (denoted as $\bar{r}_{1}$ ) and the corresponding capacity loss (denoted as $\bar{\varepsilon}$ ). Nine different simulation setups have been tested. The changes relative to the default simulation setup are indicated in the corresponding table. The results corroborate the theoretical claims and illustrate the advantages of the developed algorithms. All our schemes are able to satisfy the constraints considered in each of the schemes. The results confirm that the long-term constraints achieve a better objective than their short-term counterparts (S2-S4 vs. S5-S7). Moreover, in several cases both long-term cognitive constraints are active (S5). This never happens when the cognitive constraints have to be satisfied in the short-term (S7).

Although the space limitations prevent us from presenting additional numerical simulations, the codes used to run the simulations are available upon request.

\section{Conclusions}

This paper investigated the design of adaptive RA schemes for underlay cognitive radio scenarios with multiple primary and SUs operating over time-varying channels. One of the most critical issues in cognitive radios is how SUs coexist with (limit the interference to) PUs. Among the different metrics considered in the paper, the most important is the guarantee on the long-term capacity loss on the PUs. Guaranteeing a certain rate for PUs is typically challenging because the presence of interfering powers render the optimization non-convex. For the operating conditions considered in the paper we showed that two important facts hold. The first one is that the optimization 
problem which gives rise to the resource allocation has zeroduality gap, so that Lagrangian relaxation can be used without losing optimality. The second one is that in the dual domain the non-convex problem can be decoupled (separated) across channels and users. The latter implies that the optimization needs to be carried out only over a scalar variable, opening the door to implementation of efficient search algorithms. It was shown that the optimal resource allocation amounts to maximize a scalar quality link functional which weights: the quality of the secondary links (rate reward minus power cost) and the damage to the PUs (interfering power cost plus rate loss). The terms in the quality link functional depend on the instantaneous CSI and on several Lagrange multipliers (whose value depended on the long-term behavior of the system and the requirements of the primary and secondary networks). Extensions to scenarios where the PUs are not always active or the CSI contains imperfections were briefly discussed.

\section{REFERENCES}

[1] D. Bertsekas, A. Nedic, and A. E. Ozdaglar, Convex Analysis and Optimization, Athena Scientific, 2003.

[2] A. Goldsmith, Wireless Communications, Cambridge Univ. Press, 2005.

[3] S. Haykin, "Cognitive Radio: Brain-Empowered Wireless Communications," IEEE J. on Selected Areas in Commun., vol. 23, no. 2, pp. 201220, Feb. 2005.

[4] A. G. Marques, X. Wang, and G. B. Giannakis, "Optimal stochastic dual resource allocation for cognitive radios based on quantized CSI", IEEE Proc. of Intl. Conf. on Acoustics, Speech and Signal Process., Las Vegas, NV, Mar. 30 - Apr. 4, 2008

[5] A. Ribeiro and G. B. Giannakis, "Separation Principles in Wireless Networking," IEEE Trans. on Info. Theory, vol. 56, no. 9, pp. 44884505, Sep. 2010.

[6] R. Urgaonkar and M. Neely, "Opportunistic scheduling with reliability guarantees in cognitive radio networks," IEEE Trans. Mobile Comp., vol. 8, no. 6, pp.766-777, Jun. 2009.

[7] X. Wang, "Jointly Optimal Sensing Selection and Power Allocation for Cognitive Communications," Proc. of Globecom Conf., Miami, FL, Dec. 6-10, 2010.

[8] A. Goldsmith, S. A. Jafar, I. Maric and S. Srinivasa, "Breaking spectrum gridlock with cognitive radios: An information theoretic perspective," Proc. of the IEEE, vol. 97, no. 5, pp. 894-914, May 2009.

[9] X. Kang, Y.-C. Liang, A. Nallanathan, H. K. Garg, and R. Zhang, "Optimal power allocation for fading channels in cognitive radio networks: Ergodic capacity and outage capacity," IEEE Trans. Wireless Commun., vol. 8, no. 2, pp. 940-950, Feb. 2009.

[10] Q. Zhao and B. M. Sadler, "A survey of dynamic spectrum access," IEEE Signal Process. Mag., vol. 24, no. 3, pp. 79-89, May 2007.

[11] R. Zhang, Y.-C. Liang, and S. Cui, "Dynamic Resource Allocation in Cognite Radio Networks: A convex optimization perspective," IEEE Signal Process. Mag., vol. 27, no. 5, pp. 102-114, May 2010.

[12] R. Zhang, "On peak versus average interference power constraints for protecting primary users in cognitive radio networks," IEEE Trans. Wireless Commun., vol. 8, no. 4, pp. 2112-2120, Apr. 2009.

[13] A. G. Marques, G. B. Giannakis, and J. Ramos, "Optimizing Orthogonal Multiple Access based on Quantized Channel State Information," IEEE Trans. on Signal Process., vol. 59, no. 10, pp. 5023 - 5038, Oct. 2011.

[14] A. G. Marques, L. M. Lopez-Ramos, G. B. Giannakis, J. Ramos, and A. Caamano, "Optimal Cross-Layer Resource Allocation in Cellular Networks Using Channel and Queue State Information," IEEE Trans. on Vehic. Tech. (submitted, available upon request).

[15] J.A. Ayala Solares, Z. Rezki, and M.S. Alouini, "Optimal Power Allocation of a Sensor Node Under Different Rate Constraints," IEEE Proc. of Intl. Conf. on Commun., Otawa, Canada, 2012.

[16] X. Gong, S. Vorobyov, C. Tellambura, "Joint bandwidth and power allocation in cognitive radio networks under fading channels", IEEE Proc. of Intl. Conf. on Acoustics, Speech and Signal Process., Prague, Czech Rep., May. 22- 27, 2011.

[17] A. G. Marques, G. B. Giannakis, L. M. Lopez-Ramos, and J. Ramos, "Resource Allocation for Interweave and Underlay CRs under Probability-of-Interference Constraints", IEEE J. Sel. Areas Commun. (submitted, available upon request).
TABLE I: Test cases 1-3.

\begin{tabular}{|c|c|c|c|c|c|c|c|}
\hline \multicolumn{8}{|c|}{ Case 1: Default case } \\
\hline Res. \Sch. & S1 & $\mathrm{S} 2$ & S3 & $\mathrm{S} 4$ & $\overline{\mathrm{S} 5}$ & S6 & S7 \\
\hline $\bar{p}_{2}$ & 1.0 & 1.0 & 1.0 & 1.0 & 1.0 & 1.0 & 1.0 \\
\hline $\bar{c}_{2}$ & 13.6 & 13.2 & 13.4 & 13.2 & 12.5 & 12.5 & 12.5 \\
\hline $\bar{p}_{1}$ & 0.5 & 0.3 & 0.36 & 0.3 & 0.23 & 0.23 & 0.23 \\
\hline $\bar{r}_{1}$ & 3 & 3.1 & 3.1 & 3.1 & 3.2 & 3.2 & 3.2 \\
\hline $\bar{\varepsilon}$ & 0.13 & 0.09 & 0.1 & 0.09 & 0.076 & 0.077 & 0.076 \\
\hline \multicolumn{8}{|c|}{ Case 2: $\mathbb{E}\left[h_{k, 1}^{m}\right]=10 \mathrm{~dB}$} \\
\hline Res. \Sch. & S1 & $\mathrm{S} 2$ & S3 & $\mathrm{S} 4$ & S5 & S6 & S7 \\
\hline $\bar{p}_{2}$ & 1.0 & 1.0 & 1.0 & 1.0 & 1.0 & 1.0 & 1.0 \\
\hline $\bar{c}_{2}$ & 13.6 & 7.62 & 8.37 & 7.61 & 6.35 & 6.42 & 6.34 \\
\hline $\bar{p}_{1}$ & 4.7 & 0.3 & 0.65 & 0.3 & 0.3 & 0.3 & 0.3 \\
\hline $\bar{r}_{1}$ & 1.9 & 3.2 & 3.1 & 3.2 & 3.1 & 3.1 & 3.1 \\
\hline $\bar{\varepsilon}$ & 0.45 & 0.084 & 0.1 & 0.084 & 0.097 & 0.098 & 0.097 \\
\hline \multicolumn{8}{|c|}{ Case 3: $\gamma_{k}=13 \mathrm{~dB}$} \\
\hline Res. \Sch. & S1 & $\mathrm{S} 2$ & S3 & $\mathrm{S} 4$ & S5 & S6 & S7 \\
\hline $\bar{p}_{2}$ & 1.0 & 1.0 & 1.0 & 1.0 & 1.0 & 1.0 & 1.0 \\
\hline $\bar{c}_{2}$ & 13.6 & 13.2 & 13.5 & 13.2 & 12.5 & 12.8 & 12.5 \\
\hline $\bar{p}_{1}$ & 0.49 & 0.3 & 0.46 & 0.3 & 0.23 & 0.27 & 0.23 \\
\hline $\bar{r}_{1}$ & 3.9 & 4.1 & 4 & 4.1 & 4.1 & 4.1 & 4.1 \\
\hline $\bar{\varepsilon}$ & 0.11 & 0.075 & 0.1 & 0.075 & 0.063 & 0.072 & 0.063 \\
\hline
\end{tabular}

TABLE II: Test cases 4-6.

\begin{tabular}{|c|c|c|c|c|c|c|c|}
\hline \multicolumn{8}{|c|}{ Case 4: $\gamma_{k}=7 \mathrm{~dB}$} \\
\hline Res. \Sch. & S1 & $\mathrm{S} 2$ & $\overline{S 3}$ & $\overline{S 4}$ & S5 & S6 & S7 \\
\hline $\bar{p}_{2}$ & 1.0 & 1.0 & 1.0 & 1.0 & 1.0 & 1.0 & 1.0 \\
\hline $\bar{c}_{2}$ & 13.6 & 13.2 & 13.1 & 13.1 & 12.5 & 12.1 & 12.1 \\
\hline $\bar{p}_{1}$ & 0.49 & 0.3 & 0.28 & 0.28 & 0.23 & 0.2 & 0.2 \\
\hline $\bar{r}_{1}$ & 2.2 & 2.3 & 2.3 & 2.3 & 2.3 & 2.4 & 2.4 \\
\hline $\bar{\varepsilon}$ & 0.15 & 0.11 & 0.1 & 0.1 & 0.092 & 0.082 & 0.081 \\
\hline \multicolumn{8}{|c|}{ Case 5: $\varepsilon_{k}=0.05$} \\
\hline Res. \Sch. & S1 & S2 & S3 & S4 & S5 & S6 & S7 \\
\hline $\bar{p}_{2}$ & 1.0 & 1.0 & 1.0 & 1.0 & 1.0 & 1.0 & 1.0 \\
\hline $\bar{c}_{2}$ & 13.6 & 13.2 & 11.8 & 11.7 & 11.7 & 10.9 & 10.9 \\
\hline $\bar{p}_{1}$ & 0.5 & 0.3 & 0.14 & 0.14 & 0.14 & 0.12 & 0.12 \\
\hline $\bar{r}_{1}$ & 3 & 3.1 & 3.3 & 3.3 & 3.3 & 3.3 & 3.3 \\
\hline $\bar{\varepsilon}$ & 0.13 & 0.09 & 0.047 & 0.047 & 0.046 & 0.041 & 0.041 \\
\hline \multicolumn{8}{|c|}{ Case 6: $\varepsilon_{k}=0.02$} \\
\hline Res. \Sch. & S1 & S2 & $\mathrm{S} 3$ & S4 & S5 & S6 & S7 \\
\hline $\bar{p}_{2}$ & 1.0 & 1.0 & 1.0 & 1.0 & 1.0 & 1.0 & 1.0 \\
\hline $\bar{c}_{2}$ & 13.6 & 13.2 & 8.49 & 8.5 & 8.47 & 7.58 & 7.58 \\
\hline $\bar{p}_{1}$ & 0.5 & 0.3 & 0.042 & 0.041 & 0.041 & 0.032 & 0.032 \\
\hline $\bar{r}_{1}$ & 3 & 3.1 & 3.4 & 3.4 & 3.4 & 3.4 & 3.4 \\
\hline $\bar{\varepsilon}$ & 0.13 & 0.09 & 0.015 & 0.015 & 0.015 & 0.012 & 0.012 \\
\hline
\end{tabular}

TABLE III: Test cases 7-9.

\begin{tabular}{|c|c|c|c|c|c|c|c|}
\hline Res. \Sch. & S1 & S2 & S3 & S4 & S5 & S6 & S7 \\
\hline $\bar{p}_{2}$ & 1.0 & 1.0 & 1.0 & 1.0 & 1.0 & 1.0 & 1.0 \\
\hline $\bar{c}_{2}$ & 13.6 & 11.9 & 13.1 & 12 & 11.1 & 11.9 & 11.1 \\
\hline $\bar{p}_{1}$ & 0.5 & 0.15 & 0.28 & 0.16 & 0.13 & 0.16 & 0.13 \\
\hline $\bar{r}_{1}$ & 2.2 & 2.4 & 2.3 & 2.4 & 2.4 & 2.4 & 2.4 \\
\hline $\bar{\varepsilon}$ & 0.15 & 0.061 & 0.1 & 0.065 & 0.057 & 0.066 & 0.057 \\
\hline \multicolumn{8}{|c|}{ Case 8: $\mathbb{E}\left[h_{k, 1}^{m}\right]=10 \mathrm{~dB}, \varepsilon_{k}=0.05, \check{p}_{k, 1}=1$} \\
\hline Res. \Sch. & S1 & S2 & S3 & S4 & S5 & S6 & S7 \\
\hline $\bar{p}_{2}$ & 1.0 & 1.0 & 1.0 & 1.0 & 1.0 & 0.8 & 0.8 \\
\hline $\bar{c}_{2}$ & 13.6 & 10.9 & 6.11 & 6.13 & 6.11 & 4 & 3.99 \\
\hline $\bar{p}_{1}$ & 4.6 & 1 & 0.19 & 0.2 & 0.17 & 0.14 & 0.14 \\
\hline $\bar{r}_{1}$ & 1.9 & 2.7 & 3.3 & 3.3 & 3.3 & 3.3 & 3.3 \\
\hline $\bar{\varepsilon}$ & 0.45 & 0.22 & 0.05 & 0.05 & 0.05 & 0.048 & 0.048 \\
\hline \multicolumn{8}{|c|}{ Case 9: $\mathbb{E}\left[h_{k, 1}^{m}\right]=10 \mathrm{~dB}, \varepsilon_{k}=0.05, \check{p}_{k, 1}=1, \check{p}_{k, 2}^{m}=1$} \\
\hline Res. \Sch. & S1 & $\mathrm{S} 2$ & S3 & S4 & S5 & S6 & S7 \\
\hline$\overline{\bar{p}_{2}}$ & 1.0 & 1.0 & 1.0 & 0.6 & 0.6 & 0.4 & 0.4 \\
\hline $\bar{c}_{2}$ & 13.5 & 10.9 & 6.11 & 5.3 & 5.32 & 3.41 & 3.44 \\
\hline $\bar{p}_{1}$ & 4.6 & 1 & 0.18 & 0.2 & 0.18 & 0.14 & 0.14 \\
\hline $\bar{r}_{1}$ & 1.9 & 2.7 & 3.3 & 3.3 & 3.3 & 3.3 & 3.3 \\
\hline $\bar{\varepsilon}$ & 0.45 & 0.22 & 0.05 & 0.05 & 0.05 & 0.048 & 0.048 \\
\hline
\end{tabular}

